\title{
Pricing Strategy for End-of-Life Electrical and Electronic Products with Remanufacturing under Joint Recovery Mode
}

\author{
Wang Jinmei ${ }^{1, *}$, Ou Shumei ${ }^{1}$ and Li Shuling ${ }^{2}$ \\ ${ }^{1}$ School of Economics and Management, Fuzhou University, Fuzhou 350108, China \\ ${ }^{2}$ Business School, The University of Sydney, Sydney NSW 2006, Australia
}

Received 14 June 2017; Accepted 1 October 2017

\begin{abstract}
Implementing a closed-loop supply chain in the appliance industry offers great practical significance given the characteristics of remanufacturing the decomposed parts of end-of-life electrical and electronic products. However, only few studies have analyzed the integration of a closed-loop supply chain for such products. To characterize this supply chain, find a suitable recovery mode, and determine its pricing strategy, a closed-loop supply chain with a manufacturer, retailer, third party recycling center, and consumers was designed. Afterward, a new joint recovery mode conducted by a retailer and third party recycling center was determined, and some pricing decision models under different mechanisms were built. Finally, corresponding operations management strategies were proposed by comparing pricing decision strategies under the cooperative and non-cooperative mechanisms based on game theory and optimization theory. The results demonstrate that the optimal selling price of each member enterprise under different mechanisms is the decreasing function of the sensitivity coefficient of consumers to selling price. Meanwhile, the overall profit of a closed-loop supply chain under different mechanisms gradually increases along with the proportion of remanufactured parts in end-of-life electrical and electronic products that can be directly used in remanufacturing. Moreover, Adopting different remanufacturing strategies under various operating mechanisms can differentiate the levels of profit. The proposed joint recovery mode is generally supportive of transaction cost theory, and the pricing strategy under different mechanisms is expected to have practical implications for enterprises to improve their operation benefits.
\end{abstract}

Keywords: End-of-life electrical and electronic products, Recycling and remanufacturing, Joint recovery mode, Closed-loop supply chain, Differential pricing

\section{Introduction}

With respect to the strict environment protection and awareness of worldwide resource shortage, recycling and remanufacturing are effective techniques for achieving economically and environmentally sustainable goals. The traditional production-consumption mode can be simply illustrated as an open "resources-production-consumptionwaste" loop, but a closed-loop supply chain is formed when the usual forward supply chain management becomes associated with the recycling process. Recycling, remanufacturing, and selling remanufactured products are three important components of a closed-loop supply chain [1]. As the remanufacturing of end-of-life electrical and electronic products belongs to the category of technology, research on closed-loop supply chains remains focused on the recycling and selling of remanufactured products. In other words, the effective recycling mode and the optimal price problem are the core issues of a closed-loop supply chain.

In the existing literatures, end-of-life electrical and electronic products are often recycled using either the reverse self-recycling mode being conducted by manufacturers or the traditional recycling mode being

\footnotetext{
*E-mail address: rucy03@163.com

ISSN: $1791-2377$ @ 2017 Eastern Macedonia and Thrace Institute of Technology. All rights reserved. doi:10.25103/jestr.105.09
}

conducted separately by distributors, retailers, and third party recycling centers [2-4]. Many studies have examined the centralized and decentralized pricing strategy by using the Stackelberg game model [5-8].

However, when recycling end-of-life electrical and electronic products, the consumers, retailers, distributors, manufacturers, and transport agents cooperate during the sorting, processing, and other stages of the recycling process. Therefore, the traditional separate recycling modes pose many risks, such as easily increasing the ex ante and ex post transaction costs, reducing recovery efficiency, and hindering operations integration. Given the specific characteristics of end-of-life electrical and electronic products in China, an appropriate recycling mode and optimal pricing strategy must be devised to coordinate the industry chain, promote the comprehensive utilization of resources, and develop a circular economy.

Based on the above analysis, this study examines a core problem in the recovery of end-of-life electrical and electronic products in a closed-loop supply chain. The pricing decision and corresponding strategies are also considered.

\section{State of the art}

Many studies have examined the reverse network design, recycling mode, coordinated operation, and pricing strategy for end-of-life electrical and electronic products in a closed- 
loop supply chain. Savaskan et al. proposed a mode in which the retailer is responsible for the recovery, and this mode is more efficient than reverse logistics self-network being conducted by the manufacturer or reverse logistics outsourcing [2]. Wang and Da argued that recycling by retailers can greatly benefit the consumers because the new products manufactured and remanufactured by a third party recycling center are more expensive than those manufactured by retailers [3]. Luu et al. studied the reverse logistics network planning for electronic products by taking the minimum system total cost as the objective function [9]. Masoudipour et al. analyzed the reverse logistics recovery rate model, which shows that a higher recovery rate corresponds to a greater profit [10]. These studies assumed that end-of-life electrical and electronic products are recycled separately by retailers and third party recycling centers as well as failed to consider the possibility of implementing a joint recovery mode. Many studies also focused on the coordination of a closed-loop supply chain. Zhong et al. presented a system dynamics model to encourage residents to participate in the dismantling of environmental protection; their simulation results showed that the illegal recovery returns could be effectively reduced by applying several measures, such as improving the recycling price and service quality by formal recycling operators and increasing the expenditures of the government for environmental protection publicity 11]. Beril and Wei designed a coordinated pricing mechanism that covered a variety of cost allocation techniques [12]. Wei and Wang modified the individual parameters of a closed-loop supply chain model to resemble the actual situation and then proposed a revenue sharing contract coordination mechanism [13]. Zhang et al. developed a coordinated pricing strategy based on the remanufacturing priority for a closed-loop supply chain in which the retailer is responsible for recycling end-of-life goods [14]. Du et al. showed that the subsidies provided by the government for recycling would effectively encourage enterprises and the public to participate in reverse logistics [15]. Liu et al. established a model for recycling end-of-life electrical and electronic products by applying the system dynamics principle [16]. Based on the extended producer responsibility system, Hsuan Hong and Jhih-Sian determined the optimal government subsidy rate and the product recovery compensation payment coefficient by applying game theory [17]. Jafar et al. suggested that price discount contracts could be used to achieve supply chain coordination [18]. All these studies investigated the coordination strategies or mechanisms for end-of-life electrical and electronic products in a closed-loop supply chain, while some researchers also discussed and built recycling models for a closed-loop supply chain. However, these works only focused on traditional separate recycling modes and did not consider the specific characteristics of end-of-life electrical and electronic products and the operation integration of a closedloop supply chain.

For the pricing strategy of a closed-loop supply chain, Bao et al. formulated a differential price strategy under the assumption that product demand was influenced by price [19]. Kaya analyzed three models of the production decision problem in the process of remanufacturing under an uncertain environment [20]. Based on the assumption that demand and cost are uncertain, Wei and Zhao explored how manufacturers and retailers formulated pricing strategies through a fuzzy analysis [21]. Zhao and Chang analyzed new and remanufactured versions of the same product by using the Lagrangean relaxation and dynamic programming schemes [22]. To analyze the optimal pricing strategies for centralized and decentralized decisions, Wang and Wang established a Stackelberg game model under the nonlinear demand condition [5]. Zhang et al. studied the concentrated and decentralized discriminatory pricing strategies of new and remanufactured products based on the Stackelberg game theory method and found that the efficiency of a closed-loop supply chain based on a concentrated pricing decision was higher than that based on a decentralized pricing decision [6] Hong et al. investigated the effects of local advertising, the collection of used products, and the pricing decisions in decentralized and centralized closed-loop supply chains by using the Stackelberg game model [7]. Maiti and Giri studied the pricing problem by constructing five schemes, namely, a centralized scheme, a decentralized scheme, and three different Stackelberg games, and found that the centralized decision was the optimal pricing decision form [8]. Huang and Wang developed three hybrid remanufacturing models (i.e., M type, MD type, and MT type) for a closed-loop remanufacturing supply chain consisting of a manufacturer, a distributor, and a third party recycling enterprise, and determined the optimal pricing strategy of each member by applying the Stackelberg game model [4]. Elham and Behnam proposed a nonlinear programming model for the location-inventory-pricing decisions of a three-level closed-loop supply chain [23]. Although much work has been devoted to the pricing of a closed-loop supply chain, they all focused on a traditional recovery mode that is separately conducted by manufacturers, distributors, retailers, and third party enterprises. Only few of these works examined the joint recovery mode based on the integration of the whole recycling process despite the uniqueness of end-of-life electrical and electronic products. This research trend does not coincide with the actual demand for end-of-life electrical and electronic products in a reverse supply chain, thereby affecting the efficiency of the entire closed-loop supply chain.

By analyzing the specific characteristics and processes of end-of-life electrical and electronic products, this study proposes a new joint recovery mode that is conducted by both the retailer and the third party recycling center. The pricing decision models under different mechanisms are based on supply chain management theory, remanufacturing theory, and game theory. The properties of the models are analyzed and the optimal solutions are derived by employing effective approaches.

The reminder of this study is organized as follows. Section 3 presents the problem description, model notation, and assumptions as well as establishes the pricing decision models under different mechanisms. Section 4 presents and discusses the optimal solutions for the two models. Section 5 summarizes the conclusions.

\section{Methodology}

\subsection{Problem description}

Consider a closed-loop supply chain composed of a manufacturer, a retailer, a third party recycling center, and consumers. In the forward chain, a manufacturer that possesses manufacturing and remanufacturing production lines can produce new products directly with new components and materials, remanufacture used products, and distribute new and remanufactured products through a 
retailer. In the reverse supply chain, the end-of-life electrical and electronic products are collected by the retailer from the final consumer and are then repurchased and divided into three categories by a third party. Those parts that can be directly used for remanufacturing are recycled by the manufacturer, those parts that still have some value as materials are sold to the material suppliers, and the completely retired parts are used as landfill.

The flow chart in Figure 1 can be simplified and integrated with decision parameters, and then the pricing decision diagram under the collaboration recycling mode can be obtained (see Fig. 2).

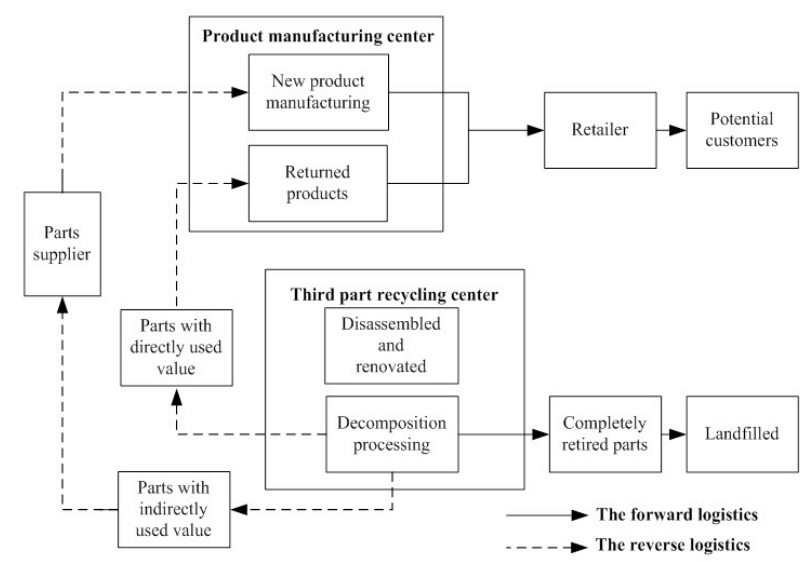

Fig.1. Graphical representation of a closed-loop supply chain

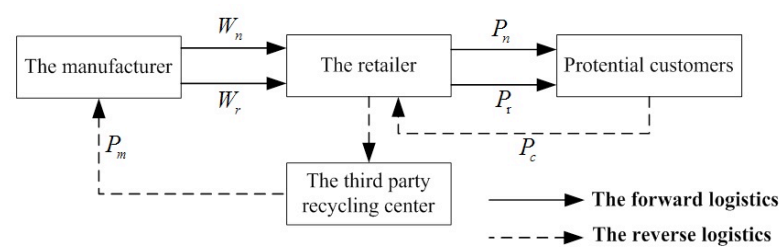

Fig. 2. Diagrammatical sketch of a pricing decision under the joint recovery mode

In the forward supply channel, the manufacturer sells the new and remanufactured products at price $W_{n}$ and $W_{r}$, and then the retailer sells two kinds of products to the potential customers at selling price $P_{n}$ and $P_{\mathrm{r}}$. In the reverse supply channel, the retailer collects the waste household appliance at price $P_{c}$ and delivers this appliance to the third party recycling center at price $P_{t}$. The third party recycling center then delivers the used products to the manufacturers at price $P_{m}$.

\subsection{Notations}

The following notations are used for developing the proposed model:

$C_{n}, C_{r}$ : the average unit cost of manufacturing new products and remanufacturing returned products, $C_{n}>C_{r}$;

$W_{n}, W_{r}$ : the unit wholesale price of new and remanufactured products, $W_{n}>W_{r}$;

$P_{n}, P_{r}$ : the unit retail price of new and remanufactured products, $P_{n}>P_{r}$;

$P_{s}:$ the average price for a retailer to acquire a used product from the consumers;
$P_{t}$ : the average recycling price for a used product acquired by a third party recycling center from a retailer;

$x$ : the recovery rate of the marginal profit of the retailer $(0<x<1)$;

$P_{m}$ : the selling price of the processed parts that can be directly used by a third party recycling center for remanufacturing;

$n_{1}, n_{2}, n_{3}$ : the proportion of returned product parts that can be directly used for manufacturing, cannot be directly used for remanufacturing, and cannot be recycled, $n_{1}+n_{2}+n_{3}=1$

$\alpha_{t}, \beta_{t}, \gamma_{t}$ : the average unit processing cost for $n_{1}, n_{2}$, and $n_{3}$ by a third party recycling center;

$A$ : marketing capacity;

$\mu$ : sensitivity of customers to the selling price;

$D_{n}, D_{r}$ : the market demand for new and remanufactured products, $D_{n}=A-\mu P_{n}$ and $D_{r}=\mu\left(P_{n}-P_{r}\right)$;

$B$ : consumer perceptions of environment protection $(B>0)$;

$k$ : elasticity coefficient of recycling price $(k>0)$;

$Q^{T}$ : the amount of recycled waste household appliances at the recycling price of $P_{s}, Q^{T}=B+k P_{s}$;

$C_{t}, C_{a}, C_{s}$ : the unit operating cost of the recycling by a third party recycling center, the forward sales by a retailer, and the reverse recovery of goods from the customers by a retailer. The unit retail price is higher than the unit wholesale price and the unit operating cost, that is, $\mathrm{P}_{\mathrm{r}}>W_{r}+C_{a}, P_{n}>W_{n}+C_{a}$;

$\delta:$ the unit price of part $n_{2}, \delta>\beta_{t}+C_{t}+P_{t}$;

$S$ : the residual value of the unsold part in a remanufactured product, $S>C_{r}+C_{t}+\alpha_{t}$;

$\pi_{m}^{T}, \pi_{r}^{T}, \pi_{t}^{T}$ : the profit function of a manufacturer, retailer, and third party recycling center under the joint recycling mode;

$\pi^{T}$ : the total system profit under the joint recycling mode.

\subsection{Assumptions}

(1) The third party recycling center has the professional ability and equipment for recycling and processing end-ofuse electrical and electronic products;

(2) each supply chain member regards its profit maximization as the goal;

(3) new and remanufactured products are priced differently due to the differences in end-user preferences;

(4) the supply chain information is common knowledge and there is no supply and demand bullwhip;

(5) the average unit cost of manufacturing new products and remanufacturing returned products is lower than the unit wholesale retail price, while the unit wholesale retail price is lower than the unit retail price, that is, $C_{n}<W_{n}<P_{n}$ and $C_{r}<W_{r}<P_{r}$;

(6) the average acquisition price of the retailer is lower than that of the third party recycling center and the selling price, that is, $P_{s}=(1-x) P_{t}, \quad P_{s}<P_{r}<P_{n}, \quad$ and $P_{c}<P_{t}<P_{m}<P_{r}<P_{n}$. 


\subsection{Model formulation}

3.4.1 Pricing decision model under the cooperative mechanism

The joint pricing decision under the cooperative mechanism means that each node enterprise in the system is a decision- making body that maximizes its overall profit as a result of its optimal decision. In this case, each node enterprise shares information with others and makes a negotiation decision. Therefore, the total profit of the system is expressed as:

$$
\begin{aligned}
& \pi=D_{n}\left(P_{n}-C_{n}-C_{a}\right)+D_{r}\left(P_{r}-C_{r}-C_{a}\right)+S\left(n_{1} Q^{T}-D_{r}\right)+n_{2} Q^{T} \delta-Q^{T}\left(P_{s}+C_{s}\right) \\
& -Q^{T}\left(P_{s}+C_{s}\right)-Q^{T}\left(P_{n}-C_{n}+C_{a}\right)-Q^{T}\left(n_{1} \alpha_{t}-n_{2} \beta_{t}-n_{3} \gamma_{t}\right)
\end{aligned}
$$

Substituting the market demand function $D_{n}=A-\mu P_{n}$,

$$
\begin{aligned}
& \pi=\left(A-\mu P_{n}\right)\left(P_{n}-C_{n}-C_{a}\right)+\mu\left(P_{n}-P_{r}\right)\left(P_{r}-C_{r}-C_{a}-S\right)+\left(B+k P_{s}\right) \\
& +\left[n_{1}\left(S-\alpha_{t}\right)+n_{2}\left(\delta+\beta_{t}\right)+n_{3} \gamma_{t}-P_{s}-C_{s}-P_{t}-C_{t}\right]
\end{aligned}
$$

\subsubsection{Pricing decision model under the non-cooperative} mechanism

In the non-cooperative mechanism, the primary purpose of each member is to acquire an optimal strategy and profit in equilibrium. The manufacturer is considered the price leader, while the retailer and third party recycling center are considered the decision followers. In the pricing process, the

$$
\pi_{m}^{T}=D_{n}\left(W_{n}-C_{n}\right)+D_{r}\left(W_{r}-C_{r}\right)+S\left(n_{1} Q^{T}-D_{r}\right)
$$

$D_{r}=\mu\left(P_{n}-P_{r}\right)$ and the recovery function $Q^{T}=B+k P_{s}$ into Eq. (1) yields:

manufacturer determines the pricing strategy, the third party recycling center takes advantage of information transparency to develop a recycling price strategy, and the retailer determines the recycling price at the end of the forward supply.

The profit function of the manufacturer can be defined as:

The profit function of the retailer can be defined as:

$$
\pi_{r}^{T}=D_{n}\left(W_{n}-C_{n}-C_{a}\right)+D_{r}\left(P_{r}-W_{r}-C_{a}\right)+Q^{T}\left(P_{t}-P_{s}-C_{s}\right)
$$

The profit function of the third party recycling center recycling can be expressed as:

$\pi_{t}^{T}=n_{1} Q^{T} P_{m}+n_{2} Q^{T} \delta-n_{1} Q^{T} \alpha_{t}-n_{2} Q^{T} \beta_{t}-n_{3} Q^{T} \gamma_{t}-\left(P_{t}+C_{t}\right)$

Substituting the market demand function $D_{n}=A-\mu P_{n}$ and $D_{r}=\mu\left(P_{n}-P_{r}\right)$, and the recycling amount function

$$
\begin{aligned}
& \pi_{m}^{T}=\left(A-\mu P_{n}\right)\left(W_{n}-C_{n}\right)+\mu\left(P_{n}-P_{r}\right)\left(W_{r}-C_{r}-S\right)+n_{1}\left(B+k P_{s}\right)\left(S-P_{m}\right) \\
& \pi_{r}^{T}=\left(A-\mu P_{n}\right)\left(W_{n}-C_{n}-C_{a}\right)+\mu\left(P_{n}-P_{r}\right)\left(P_{r}-W_{r}-C_{r}\right)+\left(B+k P_{s}\right)\left(P_{t}-P_{s}-C_{s}\right) \\
& \pi_{t}^{T}=\left(B+k P_{s}\right)\left[n_{1}\left(P_{m}-\alpha_{t}\right)+n_{1}\left(\delta-\beta_{t}\right)-n_{3} \gamma_{t}-P_{t}+C_{t}\right]
\end{aligned}
$$

The game process under symmetric informationcan be solved by the bi-level optimization model as:

$$
\max \pi_{m}^{T}=\left(A-\mu P_{n}\right)\left(W_{n}-C_{n}\right)+\mu\left(P_{n}-P_{r}\right)\left(W_{r}-C_{s}-S\right)+n_{1}\left(B+k P_{s}\right)\left(S-P_{m}\right)
$$

S.t. $\quad P_{n}=\arg \max \pi_{r}^{T}$

$P_{r}=\arg \max \pi_{r}^{T}$

$P_{s}=\arg \max \pi_{r}^{T}$ 
$P_{n}=\arg \max \pi_{t}^{T}$

\section{Result analysis and discussion}

$\left\{\begin{array}{l}P_{n}^{\prime \prime}=-\frac{C_{r}}{3}+\frac{2 C_{n}}{3}+\frac{C_{a}}{3}-\frac{S}{3}+\frac{2 A}{3} \\ P_{r}^{\prime \prime}=\frac{C_{r}}{3}+\frac{C_{n}}{3}+\frac{2 C_{a}}{3}+\frac{S}{3}+\frac{A}{3} \\ P_{s}^{\prime \prime}=\left[n_{1}\left(S-\alpha_{t}\right)+L-C_{s}\right]\left(\frac{1+3 x}{3+5 x}\right)-\frac{B(1+2 x)}{k(3+5 x)} \\ P_{t}^{\prime \prime}=\left[n_{1}\left(S-\alpha_{t}\right)+L-C_{s}\right]\left(\frac{1-3 x}{3+5 x}\right)-\frac{B(1+x)}{k(3+5 x)}\end{array}\right.$

By substituting Eq. (10) into Eq. (1), the total profit of

$\pi^{*}=\frac{1}{9}(A-2 \mu Q+\mu H)\left(\frac{2 A}{\mu}-Q-H\right)+\frac{1}{9}(A-2 \mu H+\mu Q)\left(\frac{A}{\mu}+Q-2 H\right)$

$+\frac{k}{3+5 x}\left\{\left[n_{1}\left(S-\alpha_{t}\right)+\left(L-C_{s}\right)\right](1+3 x)+\frac{B(2+3 x)}{k}\right\}$

where $Q=C_{n}+C_{a}, H=C_{r}+C_{a}+S$, and $L=n_{2}\left(\delta-\beta_{t}\right)-n_{3} \gamma_{t}-C_{t}$.

4.2 Solution for the pricing decision model under the non-cooperative mechanism

By applying the inverse induction method, the first and second order derivatives of parameters $P_{n}, P_{r}$, and $P_{s}$ in Eq.

$$
\left\{\begin{array}{l}
P_{n}=\frac{2 W_{n}}{3}-\frac{W_{r}}{3}+\frac{C_{a}}{3}+\frac{2 A}{3 \mu} \\
P_{r}=\frac{W_{n}}{3}+\frac{W_{r}}{3}+\frac{2 C_{a}}{3}+\frac{A}{3 \mu} \\
P_{s}=\frac{P_{t}}{2}-\frac{C_{s}}{2}-\frac{B}{2 k} \\
P_{t}=\frac{1}{2} n_{1}\left(P_{m}-\alpha_{t}\right)+\frac{L}{2}+\frac{B}{2 k(x-1)}
\end{array}\right.
$$

Given that the second derivatives are less than 0 , according to Most Value Property, Eq. (12) is the derivation $\pi_{m}^{T}=\left(\frac{A}{3}-\frac{2}{3} \mu W_{n}+\frac{1}{3} \mu W_{r}-\frac{1}{3} \mu C_{a}\right)\left(W_{n}-C_{n}\right)$

$+\mu\left(\frac{A}{3}+\frac{1}{3} \mu W_{n}-\frac{2}{3} \mu W_{r}-\frac{1}{3} \mu C_{a}\right)\left(W_{r}-C_{r}-S\right)$

$+\left[\frac{B}{2}+\frac{B}{4(x-1)}+\frac{1}{4} k n_{1}\left(P_{m}-\alpha_{t}\right)+\frac{1}{4} L k-\frac{1}{2} k C_{s}\right]\left[n_{1}\left(S-P_{m}\right)\right]$

where $L=n_{2}\left(\delta-\beta_{t}\right)-n_{3} \gamma_{t}-C_{t}$.

The first and second order derivatives of $\pi_{m}^{T}$ and Eq. (12) on the decision variables $W_{n}, W_{r}$, and $P_{m}$ can be

$$
\left\{\begin{array}{l}
W_{n}=\frac{C_{n}}{2}-\frac{C_{a}}{2}+\frac{A}{2 \mu} \\
W_{r}=\frac{C_{r}}{2}-\frac{C_{a}}{2}+\frac{S}{2}+\frac{A}{2 \mu} \\
P_{m}=\frac{1}{2}\left(S+\alpha_{t}\right)-\frac{1}{2 n_{1}}\left(L-2 C_{s}\right)-\frac{B}{2 k n_{1}}\left(2+\frac{1}{x-1}\right)
\end{array}\right.
$$

Given that the second derivatives are less than 0, Eq. (14) is the derivation point of the variables in Eq. (6). The
4.1 Solution for the pricing decision model under the cooperative mechanism

Under the cooperative mechanism, the pricing decision problem can be transformed into the problem of solving the minimum function. If the first derivative of Eq. (2) is 0 , then:

the closed-loop supply chain system under the cooperative mechanism is given by:

(7) as well as the first and second order derivatives of parameter $P_{t}$ in Eq. (8) can be obtained. After making the first derivative equal to 0 , Eq. (12) can be obtained as below:

point. Substituting Eq. (12) into the profit function of the manufacturer yields:

obtained. Making the first derivative equal to 0 yields:

optimal solutions of Eq. (9) are described as below: 


$$
\left\{\begin{array}{l}
W_{n}^{*}=\frac{C_{n}}{2}-\frac{C_{a}}{2}+\frac{A}{2 \mu} \\
W_{r}^{*}=\frac{C_{r}}{2}-\frac{C_{a}}{2}+\frac{S}{2}+\frac{A}{2 \mu} \\
P_{n}^{*}=\frac{C_{n}}{3}-\frac{C_{r}}{6}+\frac{C_{a}}{6}-\frac{S}{6}+\frac{5 A}{6 \mu} \\
P_{r}^{*}=\frac{C_{n}}{6}+\frac{C_{r}}{6}+\frac{C_{a}}{3}+\frac{S}{6}+\frac{2 A}{3 \mu} \\
P_{t}^{*}=\frac{n_{1}}{4}\left(S-\alpha_{t}\right)+\frac{L}{4}+\frac{C_{s}}{2}+\frac{B}{4 k(x-1)}-\frac{B}{2 k} \\
P_{s}^{*}=\frac{n_{1}}{8}\left(S-\alpha_{t}\right)+\frac{L}{8}-\frac{C_{s}}{4}+\frac{B}{8 k(x-1)}-\frac{B}{4 k} \\
P_{m}^{*}=\frac{1}{2}\left(S-\alpha_{t}\right)+\frac{1}{2 n_{1}}\left(L-2 C_{s}\right)-\frac{B}{2 k n_{1}}\left(2+\frac{1}{x-1}\right)
\end{array}\right.
$$

Substituting the optimal solution Eq. (15) into Eqs. (7),

$\pi_{m}^{T^{*}}=\frac{1}{12}(A-2 \mu Q+\mu H)\left(\frac{A}{\mu}-Q\right)+\frac{1}{12}(A+2 \mu Q-2 \mu H)\left(\frac{A}{\mu}-H\right)$ $+\frac{k}{16}\left[\left(S-\alpha_{t}\right)+\left(L-2 C_{s}\right)+\frac{B}{k(x-1)}+\frac{2 B}{k}\right]^{2}$

The profit function of the retailer is:

$$
\begin{aligned}
& \pi_{r}^{T^{*}}=\frac{1}{36}(A-2 \mu Q+\mu H)\left(\frac{2 A}{\mu}-Q-H\right)+\frac{1}{36 \mu}(A+\mu Q-2 \mu H)^{2} \\
& +\frac{k}{64}\left[\left(S-\alpha_{t}\right)+\left(L-2 C_{s}\right)+\frac{B}{k(x-1)}+\frac{2 B}{k}\right]^{2}
\end{aligned}
$$

The profit function of the third party recycling center is:

$$
\pi_{t}^{T^{*}}=\frac{k}{32}\left[n_{1}\left(S-\alpha_{t}\right)+\left(L-2 C_{s}\right)+\frac{B}{k(x-1)}+\frac{2 B}{k}\right]^{2}
$$

Thus, the profit of the entire system under joint recycling can be expressed as below:

$$
\begin{aligned}
& \pi^{T^{*}}=\pi_{m}^{T^{*}}+\pi_{r}^{T^{*}}+\pi_{t}^{T^{*}} \\
& =\frac{1}{36}(A-2 \mu Q+\mu H)\left(\frac{5 A}{\mu}-Q-H\right)+\frac{1}{36}(A+\mu Q-2 \mu H)^{2}\left(\frac{4 A}{\mu}-Q-3 H\right) \\
& +\frac{7 k}{64}\left[\left(S-\alpha_{t}\right)+\left(L-2 C_{s}\right)+\frac{B}{k(x-1)}+\frac{2 B}{k}\right]^{2}
\end{aligned}
$$

where $Q=C_{n}+C_{a}, H=C_{r}+C_{a}+S$, and $L=n_{2}\left(\delta-\beta_{t}\right)-n_{3} \gamma_{t}-C_{t}$.

Based on the solving process, $\left(W_{n}^{*}, W_{r}^{*}, P_{n}^{*}, P_{r}^{*}, P_{s}^{*}\right.$, $\left.P_{t}^{*}, P_{m}^{*}\right)$ can be derived as the optimal pricing portfolio for all node enterprises under the non-cooperative mechanism, while $\left(\pi_{m}^{T^{*}}, \pi_{r}^{T^{*}}, \pi_{t}^{T^{*}}, \pi^{T^{*}}\right)$ can be derived as the profit function of the manufacturer, retailer, third party recycling center, and entire system, respectively.
(8), and (9) yields the following profit function of the manufacturer: 
Proof of proposition 1: Given that $\frac{\partial P_{n}^{*}}{\partial \mu}=-\frac{5 A}{6 \mu^{2}}$, $\frac{\partial P_{r}^{*}}{\partial \mu}=-\frac{2 A}{3 \mu^{2}}, \frac{\partial P_{n}^{\prime \prime}}{\partial \mu}=-\frac{2 A}{3 \mu^{2}}, \frac{\partial P_{r}^{\prime \prime}}{\partial \mu}=-\frac{A}{3 \mu^{2}}$ and the market capacity $A$ is positive, the derivatives of Eq. (11) are less than 0 , that is, the optimal selling price of end-of-life electrical and electronic products $\left(P_{n}^{*}, P_{r}^{*}, P_{n}^{\prime \prime}, P_{r}^{\prime \prime}\right)$ is the decreasing function of the sensitivity coefficients of consumers to selling price $\mu$.

Proposition 2. Whether under the non-cooperative or cooperative mechanism, the overall profits of the closedloop supply chain gradually increase along with the proportion of remanufactured parts of recycled waste electrical appliances that can be directly used in remanufacturing $n_{1}$.

$$
\begin{aligned}
& \text { Proof of proposition 2: } \quad \text { For } \\
& \int \frac{\partial \pi^{T}}{\partial n_{1}}=\frac{7 k}{32}\left[n_{1}(S-\alpha t)+L-2 C s+\frac{B}{k(x-1)}+\frac{2 B}{k}\right], \quad \text { the } \\
& \left\{\frac{\partial \pi^{T *}}{\partial n_{1}}=\frac{2 k}{3+5 x}\left\{\left[n_{1}(S-\alpha t)+L-C s\right](1+3 x)+\frac{B(2+3 x)}{k}\right\},\right.
\end{aligned}
$$

remanufacturing process is meaningful in both the model assumptions and parameter descriptions. Therefore,

$$
\begin{aligned}
& n_{1}\left(S-\alpha_{t}\right)+L-2 C_{s}+\frac{B}{k(x-1)}+\frac{2 B}{k}>0 \\
& {\left[n_{1}\left(S-\alpha_{t}\right)+L-C_{s}\right](1+3 x)+\frac{B(2+3 x)}{k}>0, \text { which indicate that the }}
\end{aligned}
$$
overall profit of the closed-loop supply chain is the increasing function of the proportion of components that can be directly used in remanufacturing $n_{1}$

Proposition 3. The joint recycling and remanufacturing strategies adopted by the system members under different operating mechanisms will lead to varying levels of profit.

Proof of proposition 3: According to the comparative analysis of Eqs. (10) and (14), the optimal price combination of each node enterprise under the non-cooperative mechanism $\left(W_{n}^{*}, W_{r}^{*}, P_{n}^{*}, P_{r}^{*}, P_{s}^{*}, P_{t}^{*}, P_{m}^{*}\right)$ differs from that under the cooperative mechanism $\left(P_{n}^{\prime \prime}, P_{r}^{\prime \prime}, P_{s}^{\prime \prime}, P_{t}^{\prime \prime}\right)$. The demand function of new electrical and electronic products $D_{n}=A-\mu P_{n}$ is also different from that of remanufactured products $D_{r}=\mu\left(P_{n}-P_{r}\right)$. The difference in sales price leads to different levels of market demand, which in turn will affect the profit of the system as shown in Eqs. (14) and (18).

\subsection{Corresponding management strategies}

Based on the above results, the following operation and management strategies are recommended for the node enterprises in the closed-loop supply chain of end-of-life electrical and electronic products:

(1) To increase the overall profit of the supply chain, the node enterprises in a closed-loop supply chain must formulate strategies to weaken the sensitivity of consumers to product price. According to proposition 1, given the background of the home appliance manufacturing industry, manufacturers can reduce the substitutability of their products by implementing unique designs, such as the Freon-free refrigerators and energy-saving air conditioners of Haier. In addition, manufacturers must focus on product quality to increase business brand recognition of consumers. Retailers must familiarize themselves with the functions of related products and make a detailed interpretation of their target customers when they are placed in charge of sales.

(2) To increase the overall profit of the supply chain, each node enterprise in a closed-loop supply chain should adopt some measures to increase the ratio of components of recycled waste electrical appliances that can be directly used in remanufacturing $\left(n_{1}\right)$. According to Proposition 2, given the background of the home appliance manufacturing industry, manufacturers must consider the recyclable disassembly of certain products in the product development and design stages. A professional third party recycling center can also cooperate with manufacturers to jointly explore the dismantling scheme of products and increase the proportion of remanufactured electrical and electronic product parts.

(3) To increase the overall profit of the supply chain, the node enterprises in a closed-loop supply chain must strengthen their coordination and cooperation. According to Proposition 3, in the joint recycling and remanufacturing model, the system profits brought by the cooperative mechanism are superior to those brought by the noncooperative mechanism. Given the background of the home appliance manufacturing industry, node enterprises must strengthen their coordination and cooperation.

\section{Conclusions}

A joint recovery mode conducted by both the retailer and third party recycling center was developed to reduce the transaction costs and improve the recovery efficiency of the closed-loop supply chain for end-of-life electrical and electronic products. The differential pricing decisions under cooperative and non-cooperative mechanisms were analyzed by using game theory and optimization theory. The following conclusions could be drawn:

(1) The joint recovery mode conducted by both the retailer and the third party recycling center accords with the view of integration management, while its specific remanufacturing process embodies the features of end-of-life electrical and electronic product remanufacturing. Therefore, this joint recovery mode is the optimal recycling mode that can realize the integration of a closed-loop supply chain for end-of-life electrical and electronic products.

(2) The optimal selling price of each node enterprise differs under the non-cooperative and cooperative mechanisms, and the system profits brought by the cooperative mechanism are superior to those brought by the non-cooperative mechanism. However, both of these mechanisms are the decreasing function of the sensitivity coefficient of consumers to selling price. The overall profit of a closed-loop supply chain gradually increases along with the proportion of remanufactured parts in the recycled waste electrical appliances that can be directly used in remanufacturing.

(3) The joint recovery mode can increase the total profit of a closed-loop supply chain for end-of-life electrical and electronic products by formulating strategies for weakening the sensitivity of consumers to product price, by adopting effective measures for improving the recycling rate, or by strengthening the coordination and cooperation among enterprises.

Some recommendations for future work are also proposed. Given that the pricing decisions for end-of-life electrical and electronic product remanufacturing are influenced by many factors, the joint recovery mode only 
considers the pricing strategy with asymmetric information. Thus, the findings of this study can be extended to remanufacturing pricing decisions with asymmetric information. The stochastic factors, such as policy and market environment changes, must also be considered in future studies.

\section{Acknowledgements}

The authors are grateful for the support provided by the Soft Science Project of the Fujian Province of China (Grant No. 2017R0053).

Access article distributed under the terms of the Creative Commons Attribution Licence

\section{References}

1. Yan, R. f., Cheng, Y. H., Wang, C. X., "Strategy analysis on differential pricing in closed-loop supply chain with remanufacturing". Chinese Journal of Management Science,1, 2013, pp.44-48.

2. Savaskan,R.C.,Bhattacharya,S.,Wassenhove,V.L.N., “Closed-loop supply chain models with product remanufacturing”. Manaonggement Science,50(2), 2004, pp.239-252.

3. Wang, W. B., Da, Q. L., "The study on collection and pricing for closed-loop supply chain with retailer and the third party collecting". Journal of Industrial Engineering /Engineering Management, 24,(2),2010, PP.130-134.

4. Huang, Y. T., Wang, Z. J., "Closed-loop supply chain models with product take-back and hybrid remanufacturing under technology licensing". Journal of Cleaner Production, 142,2017, PP.39173927.

5. Wang, Y.H., Wang, X., "Research on contract coordination and price decision of closed-loop supply chain under disruption condition". Journal of Mechanical Engineering, 49(16), 2013, PP.185-192.

6. Zhang S.H., Chu Y.P., "Research on the discriminatory pricing game model of closed-loop supply chain based on remanufacturing with government restrictions".Operations Research and Management Science,23(3),2014,PP.119-126.

7. Hong, X. P., Xu, L., Du P., Wang, W.J., "Jointed advertising, pricing and collection decision in a closed-loop supply chain". International Journal of Production Economics, 167,2015, PP.1222.

8. Maiti,T., Giri,B.C., "A closed-loop supply chain under retail price and product quality dependent demand". Journal of Manufacturing Systems,37,2015,PP.624-637.

9. Luu,Q.D., Doan,T.L., Chou,S.Y., Vincent,F.Y., "Optimizing reverse logistics costs for recycling end-of-life electrical and electronic products". Expert Systems with Application,39,2012, PP.63806387.

10. Masoudipour,E., Amirian,H., Sahraeian,R., “ A novel close-loop supply chain based on the quality of returned products". Journal of cleaner production,151,2017, PP.344-355.

11. Zhong, Y.G., Qian, Y., Yin, F.F., Zhou, X.D., “ Stimulating residents to recycle waste electrical and electronic equipments: A system dynamics model". Systems Engineering-Theory \& Practice, 30(4),2010, PP.709-722.
12. Beril, T.L., Wei, D., "Cost allocation in manufacturing remanufacturing operations". Prodution and Operations Management,20(6),2011, PP.41-47.

13. Wei, Z., Wang, Y.M., "Coordination mechanism for closed-loop supply chain contract on the view of game theory". Journal of Quantitative Economics, 2,2013, PP.34-36.

14. Zhang, S.H., Zhang, J.L, Chu, Y.P., "Pricing and coordination of closed-loop supply chain based on remanufacturing priority". Journal of Systems Engineering, 28(4),2013, PP.506-513.

15. Du, M.K., Tao, Bo., Zhu Y., "Research on incentive and propulsion mechanisms of reverse logistics for recycling waste household appliances based on the three-party game". Soft Science, 28(12),2014, PP.55-59.

16. Liu, Y.Q., Xie Z.W., Gong Q.M., Liu Y.H., "Waste household appliance recycling model considering government subsidies and competition degree of recycling”. Systems Engineering, 4, 2016 , PP.117-125.

17. Hsuan Hong, I., Jhih-Sian, K., "Determining advanced recycling fees and subsidies in"E-scrap"reverse supply chains". Journal of Environmental Management,92(6),2011, PP.1495-1502.

18. Jafar, H., Kannan, G., Amin, J., "Reverse and closed-loop supply chain coordination by considering government role". Transportation Research Part D, 52,2017, PP.379-398.

19. Bao, X.Y., Tang, Z.Y., Tang, X.W., "Coordination and differential price strategy of closed-loop supply chain with product remanufacturing". Journal of Systems \& Management, 19(5),2010, PP.546-552.

20. Kaya,O., "Incentive and production decisions for remanufacturing operations". European Journal of Operational Research,201(2),2010, PP.442-453.

21. Wei, J., Zhao, J., "Pricing decisions with retail competition in a fuzzy closed-loop supply chain". Expert Systems with Applications,38(9),2011, PP.11209-11216.

22. Zhao, J.M., Chang, C.I., "Dynamic pricing for new and remanufactured products in a closed-loop supply chain". International Journal of Production Economics,146,2013, PP.153160.

23. Elham, A., Behnam, V., "A location-inventory-pricing model in a closed-loop supply chain network with correlated demands and shortages under a periodic review system". Computer and Chemical Engineering,101,2017, PP.148-166. 